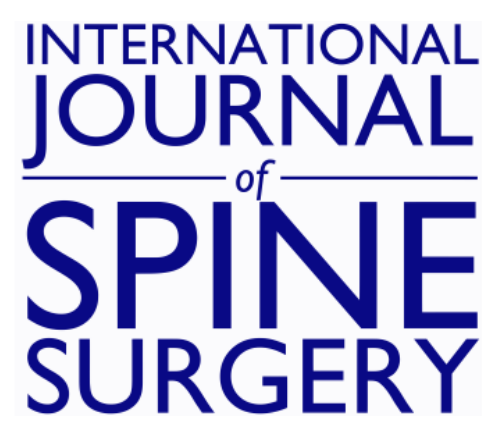

\title{
Locally Delivered Ascorbic Acid and $\beta$-Glycerophosphate Augment Local Bone Graft in a Murine Model of 2-Level Posterior Spinal Fusion
}

Joshua Vic Chen, Katie Lee, Kyle Tillinghast, Bernard Halloran and Alan B.C. Dang

Int J Spine Surg 2021, 15 (5) 921-928

doi: https://doi.org/10.14444/8120

http://ijssurgery.com/content/15/5/921

This information is current as of April 26, 2023.

Email Alerts Receive free email-alerts when new articles cite this article. Sign up at: http://ijssurgery.com/alerts 


\title{
Locally Delivered Ascorbic Acid and $\beta$-Glycerophosphate Augment Local Bone Graft in a Murine Model of 2-Level Posterior Spinal Fusion
}

\author{
JOSHUA VIC CHEN, BA, ${ }^{1}$ KATIE LEE, BA, ${ }^{1}$ KYLE TILLINGHAST, MD, ${ }^{1}$ BERNARD HALLORAN, PhD, ${ }^{2}$ \\ ALAN B.C. DANG, $\mathrm{MD}^{1,2}$ \\ ${ }^{1}$ Department of Orthopaedic Surgery, University of California, San Francisco, California, ${ }^{2}$ Orthopaedic Section, Department of Surgery, \\ San Francisco VA Health Center, San Francisco, California
}

\begin{abstract}
Background: Ascorbic acid is involved in collagen biosynthesis and upregulates alkaline phosphatase, potentially alleviating cell senescence and stimulating mesenchymal stem cell proliferation and differentiation into osteoblasts. We hypothesized locally delivered ascorbic acid and $\beta$-glycerophosphate act as a bone graft extender to increase the volume of new bone formed in a murine model of posterior lumbar fusion.

Methods: Collagen sponges were used as delivery vehicles. Sponges were prepared with primary media alone or with the addition of ascorbic acid and $\beta$-glycerophosphate. Fresh morselized bone graft from 12 donor mice was used. Twenty-four healthy male C57BL/6 mice underwent an uninstrumented posterior L3-L5 lumbar fusion. One control group received morselized bone only. A second "sponge control" group received morselized bone with the control collagen sponge. The third group received morselized bone and a collagen sponge with ascorbic acid and $\beta$ glycerophosphate. Three months postoperatively, the lumbar spine underwent high-resolution micro-computed tomography for analysis of bone formation, density, and bridging fusion.

Results: Animals receiving ascorbic acid and $\beta$-glycerophosphate had a statistically significant increase in corrected bone volume compared with control and sponge groups, with a $56.3 \%$ and $25.4 \%$ increase, respectively. Mineralized bone fraction was statistically significantly decreased for animals in the ascorbic acid group compared with control and sponge groups. There was no significant difference in fusion rate between test groups.

Conclusions: Locally delivered ascorbic acid and $\beta$-glycerophosphate in a murine model of posterior spinal fusion yielded statistically significant increases in new bone formation in the lumbar spine but statistically significant decreases in mineralized bone fraction. Differences in fusion rate were not statistically significant.

Clinical Relevance: This study provides early data suggesting that delivery of ascorbic acid to a spinal fusion site may be beneficial but does not yet establish an indication for clinical use. Further studies are needed to determine optimal dose and delivery of ascorbic acid.

Lumbar Spine

Keywords: ascorbic acid, $\beta$-glycerophosphate, bone graft enhancer, spinal fusion, bone mineral density
\end{abstract}

\section{INTRODUCTION}

Pseudarthrosis is a devastating complication, with patients facing pain, disability, and unemployment. More than $\$ 3$ billion is lost annually in direct and indirect costs due to failures to heal or form bone, such as the case with spinal fusion. ${ }^{1-3}$ An additional $\$ 2$ billion is spent on products to augment iliac crest autograft and local bone graft with bone graft extenders or substitutes, such as demineralized bone matrix, ceramics, and bone morphogenetic proteins. $^{4,5}$ More than 400000 spinal fusions are performed in the United States each year, ${ }^{6}$ and pseudarthrosis occurs in upwards of $18 \%$ of patients, ${ }^{1}$ with an average added cost of $\$ 41,631$ for treating pseudarthrosis with a second surgery. ${ }^{6}$ The currently accepted model of posterolateral spinal fusion model in rats involves augmentation with recombinant human bone morphogenetic protein-2 (rhBMP-2); although clinically efficacious, some common risks of the use of rhBMP-2 in patients include heterotopic bone formation and lifethreatening soft tissue swelling, resulting in airway obstruction in the setting of anterior cervical fusion. ${ }^{7,8}$ Therefore, the development of a newer, lower-cost, noninferior method for bone healing and formation with fewer complications is needed. 
Ascorbic acid, or vitamin $\mathrm{C}$, and its role in bone synthesis have been studied since the discovery of scurvy, ${ }^{9}$ and it has been involved in the collagen biosynthetic pathway ${ }^{10}$ and upregulates alkaline phosphatase. $^{11}$ It may alleviate cell senescence, ${ }^{12}$ stimulate mesenchymal stem cell proliferation, ${ }^{13}$ and has been shown to help differentiate mesenchymal stem cells into osteoblasts. ${ }^{14,15}$ Furthermore, ascorbic acid can be used along with cultureexpanded bone marrow stromal cells to generate robust bone. ${ }^{9,16,17}$

Given that topical antibiotics are locally delivered to reduce infection in spine surgery ${ }^{18}$ with appropriate risk to benefit ratio, ${ }^{19,20}$ we hypothesized that locally delivered ascorbic acid and $\beta$-glycerophosphate could increase bone formation and spinal fusion in a murine model of posterior lumbar fusion. As with topical antibiotics, local delivery allows higher tissue concentrations than oral dosing (given the laxative effect of high-dose oral ascorbic acid). We hypothesized that local delivery of Lascorbic acid and $\beta$-glycerophosphate through soaked Gelfoam hemostatic sponges would act as a bone graft enhancer in a murine model of posterior spinal fusion to increase bone formation.

\section{METHODS}

\section{Collagen Sponge Preparation}

Collagen sponges were used as a delivery vehicle for ascorbic acid. Commercially available hemostatic collagen sponges (Gelfoam, Pfizer, New York, New York) were sterilely cut using a disposable No. 11 blade scalpel into $1 \mathrm{~mm}^{3}$ blocks, measured by a sterile ruler, and performed under magnification. One hour prior to surgery, sponges were prepared with either primary media alone (Dulbecco modified Eagle medium:F12/fetal bovine serum [DMEM:F12/FBS]) or with ascorbic acid $(50 \mu \mathrm{g} /$ $\mathrm{mL})$ and $\beta$-glycerophosphate $(3 \mathrm{mM})$. $\beta$-Glycerophosphate provides a high local concentration of phosphate ions to assist with mineralization.

\section{Allograft Harvest and Preparation}

All animal protocols were approved by our Institutional Animal Care and Use Committee (IACUC). This study adheres to the ARRIVE guidelines. To obtain consistent volumes of fresh morselized bone graft representing both cortical and cancellous bone, 12 donor C57BL/6 mice were randomly selected from a batch of 36 healthy male mice. The remaining mice were randomly allocated to 3 test groups. Bilateral femurs were harvested, with a single femur allocated to each of 8 mice in 3 test groups. Soft tissue was removed, and the remaining bone was morselized using sterilized household nail clippers (KAI, Seki, Japan).

\section{Surgical Procedure}

A total of 24 healthy male $\mathrm{C} 57 \mathrm{BL} / 6$ mice $(6$ weeks old) underwent an uninstrumented posterior L3-L5 lumbar fusion. After isoflurane anesthesia, the lumbar region of the dorsal skin was shaved and prepped with povidone-iodine. Buprenorphine was injected subcutaneously for perioperative pain control. A $15-\mathrm{mm}$ midline incision centered over L3-L5, referencing the iliac crests as L5-L6, was made with a No. 11 blade scalpel using surgical microscopy. The paraspinal muscles covering L3 L5 were gently dissected off the lamina using the scalpel. The periosteum overlying the spinous processes and lamina were vigorously scraped by the scalpel for 20 seconds, and $1 \mathrm{~mm}^{3}$ allograft obtained from a donor mouse was placed directly on the vertebrae and was held in place by surrounding paraspinal muscles and overlying fascia upon closure of the fascial layer. The control group (control, $\mathrm{n}=8$ ) received a total of $1 \mathrm{~mm}^{3}$ morselized bone only. A second control group to define the effect of the collagen sponge/primary media (sponge, $\mathrm{n}=8$ ) received $1 \mathrm{~mm}^{3}$ morselized bone and a $1 \mathrm{~mm}^{3}$ collagen sponge prepared with primary media alone. The third group (ascorbic acid, $\mathrm{n}=8$ ) received $1 \mathrm{~mm}^{3}$ morselized bone and a $1 \mathrm{~mm}^{3}$ collagen sponge prepared with ascorbic acid and $\beta$ glycerophosphate. Collagen sponges were placed superficial to the allograft. The fascial layer was closed, holding the collagen sponges in place, and skin was closed overlying it. Bupivacaine was injected into the wound during closing as a splash block. Postoperatively, animals were given meloxicam wafers, required by our IACUC, and allowed activity without restriction. Animals were monitored daily by research and veterinary medical unit staff for humane end points, which include unrelieved pain or distress, moribund state, inability to obtain water or feed, weight loss, and decreased appetite.

\section{Micro-Computed Tomography Scans}

At 3 months postoperatively, mice were killed, and the lumbar spine was harvested en bloc with 


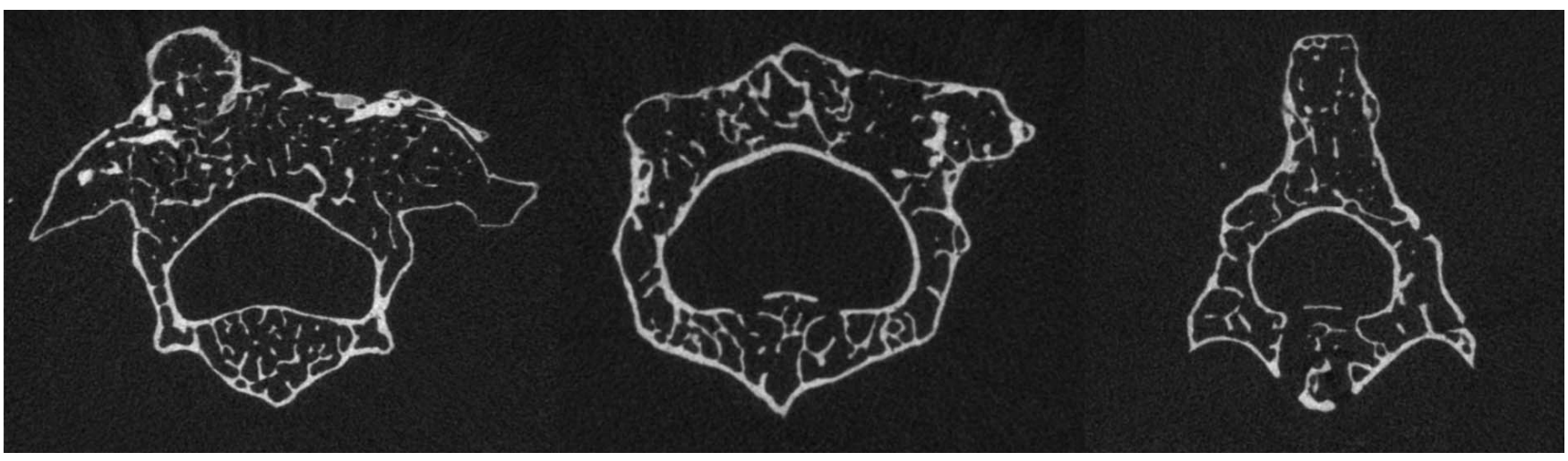

Figure 1. Axial micro-CT images (ascorbic acid group). The border between native lamina and newly formed bone is poorly defined, requiring the referencing of the anterior vertebral body to assess bone volume.

retention of the surrounding musculature. Dissected specimens were placed in $70 \%$ ethanol and scanned using a SCANCO Medical $\mu \mathrm{CT} 50$ Specimen Scanner (Zurich, Switzerland) at a slice thickness of $10 \mu \mathrm{m}$.

\section{Bone Volume and Density Quantification}

Data were quantified by a single blinded analyst to reduce variability. Reliably distinguishing between host native bone comprising the lamina and spinous processes from newly formed bone proves difficult without a comparison scan (Figure 1). This is complicated by morphologic differences between individual mice. Although in vivo whole mouse micro-computed tomography (micro-CT) scans are helpful for gross assessment of spinal fusion, imaging studies with sufficient resolution to accurately evaluate bone volume, density, and bridging bone in the murine lumbar spine require ex vivo specimen micro-CT scans that would represent lethal radiation exposures for a mouse. Therefore, we quantified bone volume using the absolute volume of bone per lumbar level and used a correction factor derived from the unoperated portion of the same level. Namely, we compared the amount of dorsal bone volume relative to the unoperated ventral bone volume, additionally allowing us to account for mouse-to-mouse variability in bone morphology. Because the dorsal bone encapsulates both newly formed bone and native lamina and spinous processes, this would decrease the measured effect of our experimental interventions. This results in a more statistically rigorous approach that makes it more difficult to achieve statistical significance.

Specifically, postprocedural micro-CT images encapsulating L3-L5 were imported into the DI-
COM viewing software OsiriX MD (Pixmeo SARL, Geneva, Switzerland). These ranges were further narrowed by only including transverse sections with continuous vertebral lamina, pedicles, and spinous processes (Figure 2). A line was drawn in a transverse section of a vertebra from the dorsalmost point of the vertebral foramina to the dorsalmost point of the vertebral body (Figure 3). This line was then bisected by a perpendicular line using the Perpendicular Lines tool (Figure 3). We defined any bone dorsal to this perpendicular line as "dorsal bone," and any bone ventral to this line as "ventral bone." This quadrant approach ensures reproducibility between specimens.

To process the large number of micro-CT images, between 60 and 160 images per level, the OsiriX region of interest (ROI)-generating algorithm was used. This algorithm predicts desired ROIs for every image based on a series of interspersed usergenerated ROIs in a given range of images. We first partitioned the images to display 1 per 20 images using the subselection tool and manually traced ROIs for the dorsal portion of the vertebrae. When the cortical bone was noncontinuous, the ROI was traced so that a line of least distance was drawn between the break (Figure 4). OsiriX uses these manual ROIs as a guide and generates ROIs for every image, which were checked by the analyst and edited to improve accuracy. OsiriX can subsequently output the collective volume and density for the dorsal portion of each vertebral level. This process was repeated for the ventral portion of the vertebrae and for each of L3-L5. A metric was created that takes the ratio of the dorsal to ventral bone volume, with the ventral bone volume serving as a level-specific correction factor. The resulting unitless ratios, corrected bone volume 


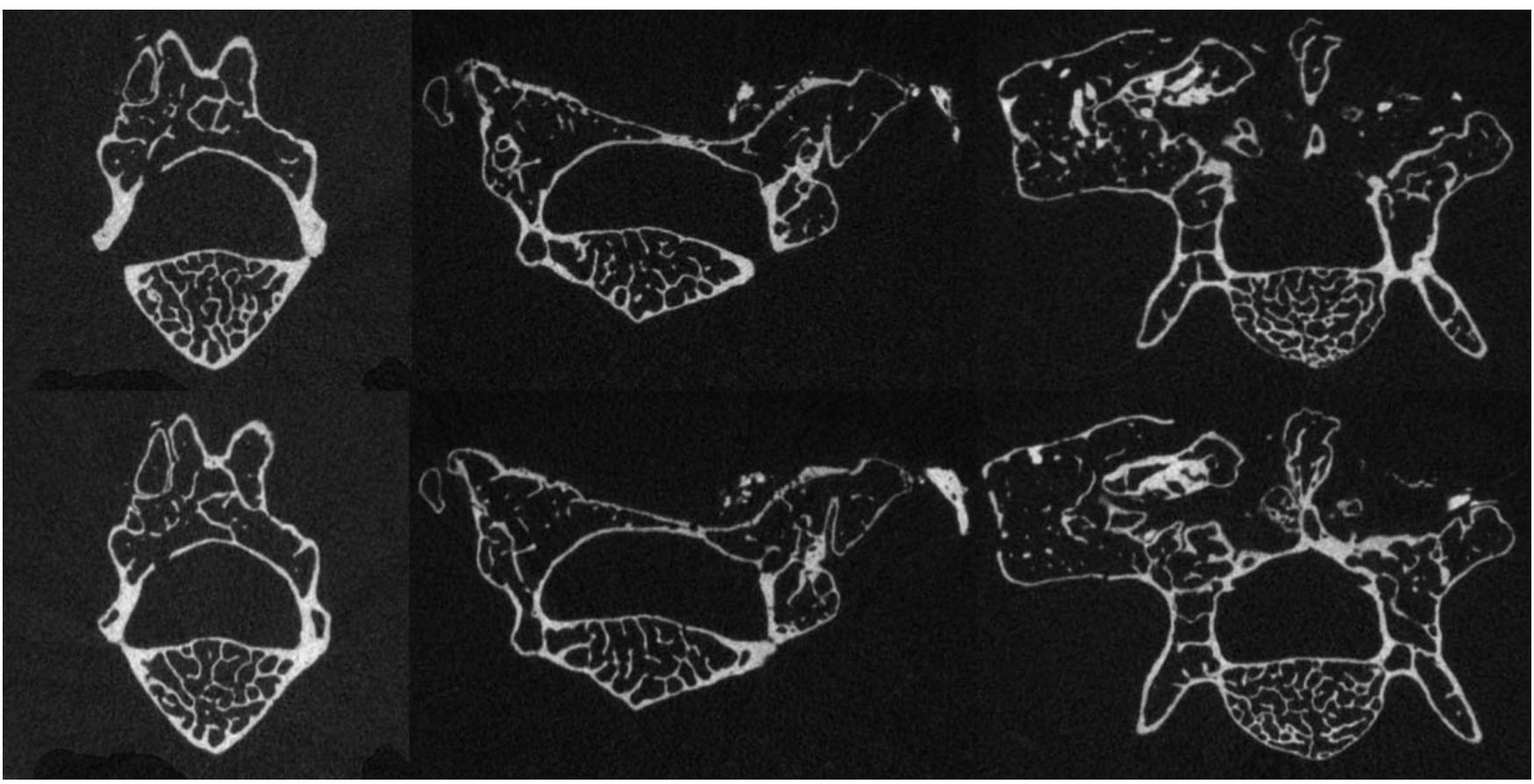

Figure 2. Analysis was performed on micro-CT images where there were continuous vertebral lamina, pedicles, and spinous processes, ensuring consistent anterior vertebral body sampling between levels. (Top) Images with noncontinuous vertebral lamina, pedicle, or spinous process. (Bottom) Images used for reference calculation.

(cBV), were calculated for each vertebra and used to compare bone volume between mice.

OsiriX additionally provides pixel density histograms of pixels encapsulated within the ROIs, and we observed a bimodal distribution of lower-density and higher-density pixels representing soft tissue and mineralized bone, respectively. Micro-CT images are calibrated to hydroxyapatite $(\mathrm{mgHA} / \mathrm{ccm})$ rather than Hounsfield units (HU); we considered pixel densities below $2000 \mathrm{mgHA} / \mathrm{ccm}$ to be soft tissue and densities above $3500 \mathrm{mgHA} / \mathrm{ccm}$ to be mineralized bone. Pixels with densities between 2000 and $3500 \mathrm{mgHA} / \mathrm{ccm}$ could not be reliably distinguished as soft tissue or mineralized bone. To compare bone mineral densities between specimens, for each lumbar level, the total number of mineralized bone pixels was divided by the sum of the number of soft tissue and mineralized bone pixels to yield a mineralized bone fraction. We investigated the mineralized bone fraction for the dorsal portion of each individual L3-L5 vertebra for each specimen. This fraction was used to compare bone mineral density between mice. This approach was used in lieu of dividing mineralized bone pixels by total ROI pixels to improve effect size by removing pixels of uncertain tissue type, with densities between 2000 and $3500 \mathrm{mgHA} / \mathrm{ccm}$.

\section{Fusion Assessment}

Full-resolution sagittal imaging sections of the lumbar spine were reviewed. Fusion was considered present or absent and was assessed at each level independently. A motion segment was considered fused if bridging bone consisting of at least $50 \%$ of the lamina of 2 adjacent segments and a continuous cortical shell from one vertebra to the adjacent vertebra were present. Three independent individuals, blinded to the test groups, assessed the specimens. Manual palpation was used in addition.

\section{Statistical Methods}

We previously determined that a sample size of 5 animals per test group was sufficient to detect significant differences in spinal fusion for rats between control and repurposed osteoarthritic chondrocytes. $^{21}$ We selected a sample size of 8 animals per test group for additional statistical power.

Statistical calculations were performed in GraphPad Prism 8 (GraphPad, San Diego, California) by a single blinded analyst. Significance was set at $P<.05$. There was a single outlier in the mineralized bone fraction data set for the sponge group identified by the interquartile range that was not omitted; these data pass the Shapiro-Wilk test 


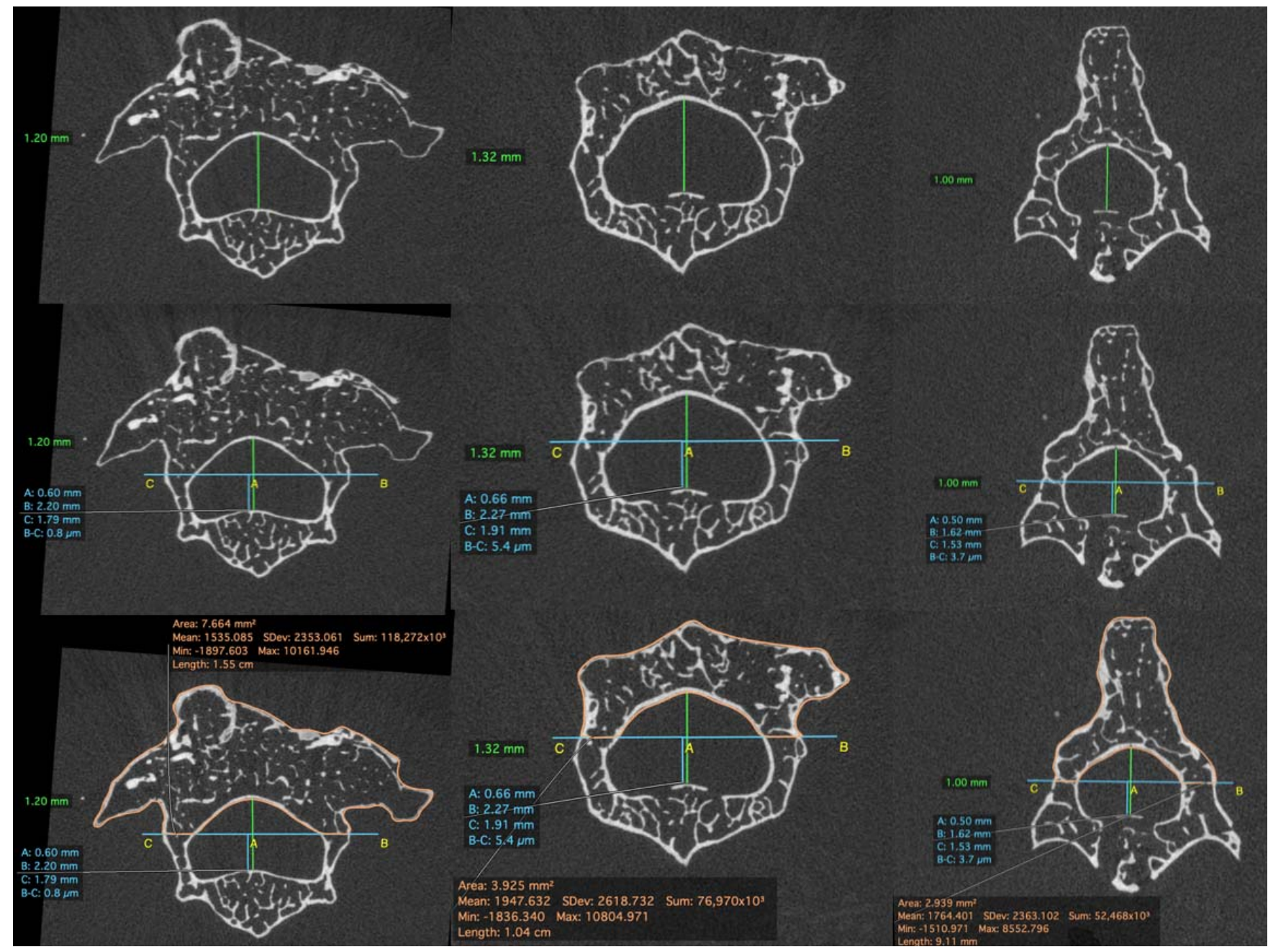

Figure 3. (Top) A line is drawn connecting the dorsal-most point of the vertebral foramen to the dorsal-most point of the vertebral body. (Middle) A bisecting perpendicular line is drawn, separating the dorsal from the ventral portion. (Bottom) Example dorsal regions of interest are traced.

of normality $(P=.0875)$. There were no additional outliers. Two-tailed Fisher exact tests were used for categoric data analysis (fusion), and Student $t$-tests were used for analysis of continuous variables (bone volume and mineralized bone fraction). Siegel-Castellan fixed-marginal multirater $\kappa$ was used to assess interrater agreement for manual palpation.

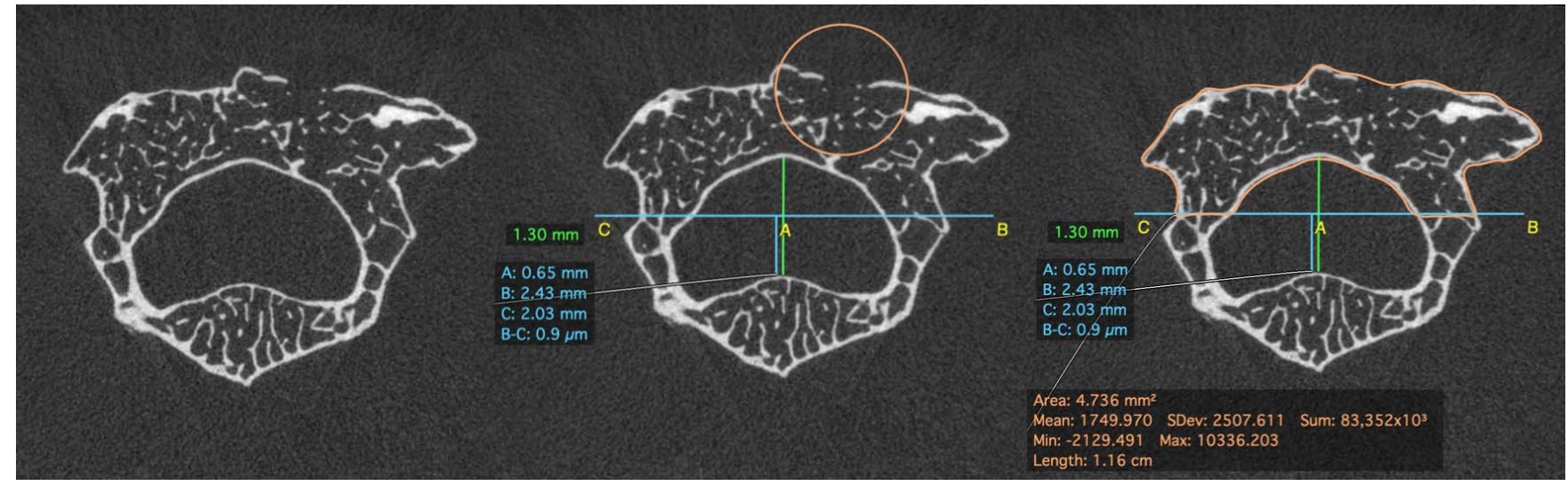

Figure 4. A line of least distance bridges noncontinuous cortical bone, drawn over a micro-computed tomography image (ascorbic acid group). The circular region of interest in the middle image identifies the noncontinuous cortical bone. 


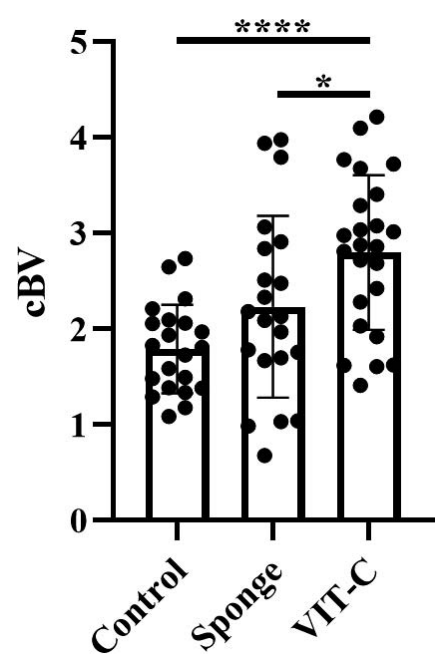

Figure 5. Corrected bone volume (cBV) is statistically significantly increased in the ascorbic acid (VIT-C) group compared with the control group $(P<.0001)$, and in the VIT-C group compared with the sponge group $(P=.0360)$. The difference in $\mathrm{CBV}$ between the sponge group and control group is nonsignificant.

\section{RESULTS}

One animal in the control group and one animal in the sponge group were killed at 4 weeks postoperatively. Mice were housed in pairs according to IACUC requirements in order to avoid social isolation from single animal housing. These 2 animals fought and both suffered irrecoverable wounds that triggered criteria for humane end point.

Animals undergoing spinal fusion with locally delivered ascorbic acid and $\beta$-glycerophosphate $(\mathrm{n}=$ 8) had a statistically significant increase in cBV compared with the control group $(\mathrm{n}=7)$, with a $56.3 \%$ increase in cBV $(P<.0001$; Figure 5). Compared to the sponge group $(\mathrm{n}=7)$, mice in the ascorbic acid group had a statistically significant increase in bone formation, with a cBV increase of $25.4 \%(P=.0360$; Figure 5$)$. Relative to controls, sponge animals had a nonsignificant increase in bone formation $(P=.0626)$. Animals in the ascorbic acid group had a statistically significant decrease in mineralized bone fraction compared with both the control group and sponge group $(P=.0421 ; P=$ 0.0078; Figure 6). Relative to controls, sponge animals had a nonsignificant increase in mineralized bone fraction $(P=.3587$; Figure 6$)$. There was no statistically significant difference in bridging bone assessed by both radiographic analysis and manual palpation between the 3 groups. A total of 13 of 32 motion segments were fused in the ascorbic acid group (40.6\% fusion), 11 of 28 in the collagen sponge-only group (39.3\% fusion), and 7 of 28 in the control group ( $25 \%$ fusion). The fusion rate of the

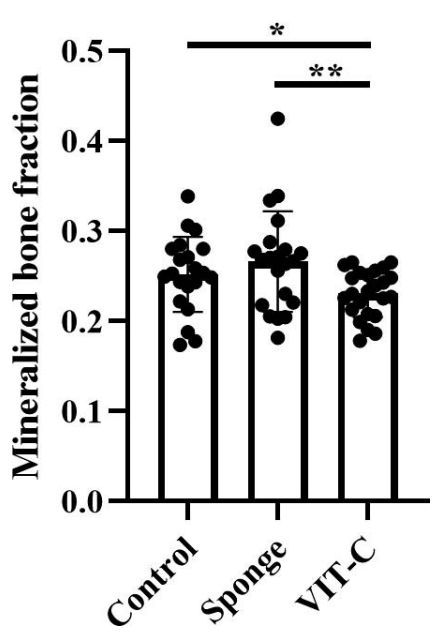

Figure 6. Mineralized bone fraction is statistically significantly decreased when comparing the VIT-C group to both the control group and sponge group ( $P$ $=.0421 ; P=.0078)$. The difference in mineralized bone fraction between the sponge group and control group is nonsignificant.

control group compared with both the sponge group and the ascorbic acid group were not significantly different $(P=.3911 ; P=.2743$; Figure 7$)$. Manual palpation had complete agreement with the CT imaging, as did the 3 blinded analysts $(\kappa=1.0)$.

\section{DISCUSSION}

Locally delivered ascorbic acid and $\beta$-glycerophosphate in a murine model of posterior spinal fusion resulted in statistically significant increases in new bone formation in the lumbar spine compared with both the control group and the group with the delivery vehicle sponge only. These differences were all statistically significant $(P<.0001$, in comparison with control). This finding is notable given the rigorous $\mathrm{cBV}$ assessment method that would be expected to weaken our statistical power and ability to detect differences. In contrast to the improvement in $\mathrm{cBV}$ seen with ascorbic acid, mineralized bone fraction was significantly deceased with vitamin $\mathrm{C}$ compared with both the control group and sponge group $(P=.0421 ; P=.0078)$. This may represent early-stage bone repair and remodeling in which there was not sufficient time to mineralize, or, conversely, mature remodeling and an appropriately decreased density of mineralized bone required for structural support as bridging bone was achieved. This requires additional investigation beyond the scope of this study. Our study was initially powered based on a previous study's sample size that observed differences in the rate of spinal fusion for rats; however, bridging fusion was not statistically significant between any test groups. ${ }^{21}$ The data sets 

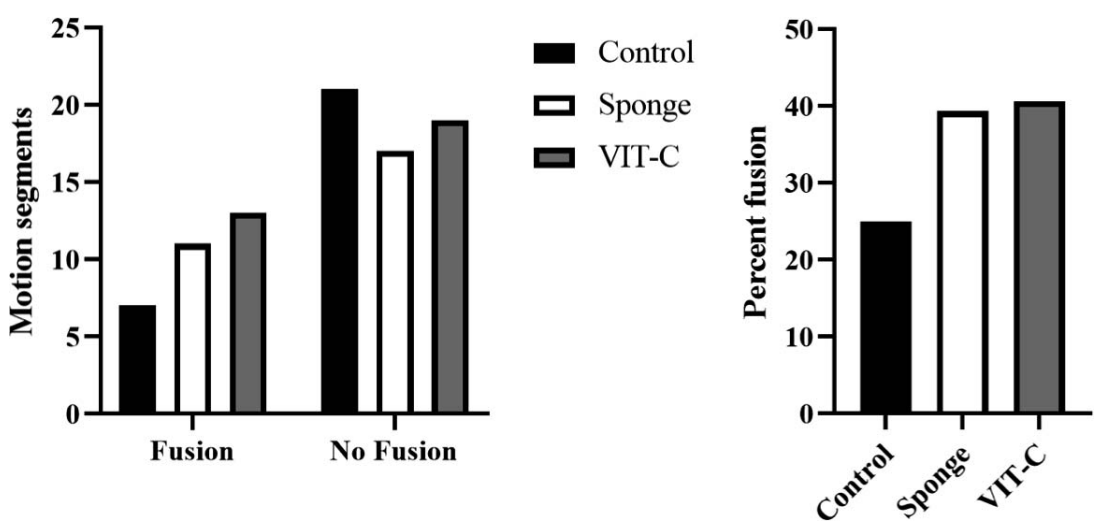

Figure 7. There is a nonsignificant difference in fusion rate between any test group. Fusion rate is nonsignificantly increased when comparing both the sponge group and ascorbic acid (VIT-C) group to the control group $(P=.3911 ; P=.2743)$.

generated and analyzed during this study may be requested from the senior author. A data use agreement may be required before release of data.

There are several limitations to this study. Importantly, our study is in mice, and results may not be directly translatable to humans. Although ascorbic acid and Gelfoam are readily available, this study does not establish an indication or contraindication for the use of locally delivered ascorbic acid and $\beta$-glycerophosphate clinically. It is possible that the administration of meloxicam, as mandated by our IACUC, inhibits spinal fusion in rodents. ${ }^{22-24}$ To understand the contribution of the sponge itself versus the role of DMEM/FBS, future studies need to assess the performance of control sponges rehydrated with DMEM without FBS. The femur contains more cortical than cancellous bone, whereas clinical bone graft typically uses more cancellous bone. Future directions include iliac crest or vertebrae as a cancellous murine donor source, histologic analysis of newly formed bone, and mechanical testing to measure segment stiffness.

Nonetheless, the strength of this study is that it is the first study to show the effect of using topical ascorbic acid in a spinal fusion model. The dose in this study $(50 \mu \mathrm{g} / \mathrm{mL})$ exceeds what can be achieved with oral dosing in humans. Over-the-counter vitamin $\mathrm{C}$ tablets are $500 \mathrm{mg}$ and are typically taken 1 to 2 times daily, resulting in plasma concentrations of $24 \mu \mathrm{g} / \mathrm{mL}{ }^{25}$ With oral administration of $3 \mathrm{~g}, 6$ times daily, serum levels peak at $38 \mu \mathrm{g} / \mathrm{mL}{ }^{25}$ Additional oral absorption is not possible because of the laxative effect associated with vitamin $\mathrm{C}$ megadoses. ${ }^{26}$ An alternative to topical delivery of ascorbic acid could include the use of intravenous ascorbic acid because plasma concentrations can reach as high as $2641 \mu \mathrm{g} / \mathrm{mL}$ with no noted toxicity. ${ }^{25}$

\section{CONCLUSION}

This study shows that small doses of locally delivered ascorbic acid and $\beta$-glycerophosphate can generate statistically significant increases in bone volume in a murine model of posterior lumbar fusion. It uses rigorous and reproducible computational analysis for the micro-CT imaging that requires larger effect sizes for statistical significance. Given the low cost of collagen hemostatic sponges and ascorbic acid, further studies may be beneficial in determining the optimal scaffold and dose of ascorbic acid, as well as individual contributions of ascorbic acid versus $\beta$-glycerophosphate.

\section{ACKNOWLEDGMENTS}

This work was supported by unrestricted funds from the University of California, San Francisco, Department of Orthopaedic Surgery and the Northern California Institute for Research and Education. This animal studies protocol was approved by the VA Medical Center, San Francisco, California Animal Studies Subcommittee (IACUC), protocol No. 15-032-01.

\section{REFERENCES}

1. Rajaee SS, Bae HW, Kanim LEA, Delamarter RB. Spinal fusion in the United States: analysis of trends from 1998 to 2008. Spine. 2012;37(1):67-76. doi:10.1097/BRS.0b013e318 $20 \mathrm{cccfb}$

2. Hustedt JW, Blizzard DJ. The controversy surrounding bone morphogenetic proteins in the spine: a review of current research. Yale J Biol Med. 2014;87(4):549-561.

3. Adogwa O, Parker S, Shau D, et al. Cost per qualityadjusted life year gained of revision fusion for lumbar pseudoarthrosis: defining the value of surgery. $J$ Spinal Disord Tech. 2015;28(3):101-105. doi:10.1097/bsd.0b013e318269cc4a

4. Spine biologics market worth $\$ 2.4$ billion by 2020 [press 
release]. Published November 24, 2015. http://www.prnewswire. com/news-releases/spine-biologics-market-worth-24-billion-by2020-553188411.html. Accessed: April 19, 2019.

5. Bone Biologics Corp. FORM 8-K. 2014. http://www.sec. gov/Archives/edgar/data/1419554/000149315214003110/ form8k.htm. Accessed: April 19, 2019.

6. Parker S, Shau D, Mendenhall S, et al. Cost per qualityadjusted life year gained of revision lumbar surgery for adjacent segment disease, pseudarthrosis, and same-level recurrent stenosis: defining the value of surgical intervention. Poster presented at: 28th Annual Meeting of the AANS/CNS Section on Disorders of the Spine and Peripheral Nerves; March 2012; Orlando, Florida.

7. Tannoury CA, An HS. Complications with the use of bone morphogenetic protein 2 (BMP-2) in spine surgery. Spine J. 2014;14(3):552-559. doi:10.1016/j.spinee.2013.08.060

8. Diwan AD, Khan SN, Cammisa FP, Sandhu HS, Lane JM. Nitric oxide modulates recombinant human bone morphogenetic protein-2-induced corticocancellous autograft incorporation: a study in rat intertransverse fusion. Eur Spine J. 2010;19(6):931-939. doi:10.1007/s00586-009-1263-7

9. Bourne GH. Vitamin C and bone. In: Bourne GH, ed. The Biochemistry and Physiology of Bone. Vol. II: Physiology and Pathology. 2nd ed. New York, NY: Academic Press; 2012:231.

10. Padh H. Vitamin C: newer insights into its biochemical functions. Nutr Rev. 1991;49(3):65-70. doi:10.1111/j.1753-4887. 1991.tb07407.x

11. Erickson C, Payne K. Inductive signals and progenitor fates during osteogenesis. In: Reis RL, Gomes ME, eds. Encyclopedia of Tissue Engineering and Regenerative Medicine. Vol. 2. New York, NY: Academic Press; 2019:395-404.

12. Esteban MA, Wang T, Qin B, et al. Vitamin C enhances the generation of mouse and human induced pluripotent stem cells. Cell Stem Cell. 2010;6(1):71-79. doi:10.1016/j.stem.2009.12.001

13. Choi KM, Seo YK, Yoon HH, et al. Effect of ascorbic acid on bone marrow-derived mesenchymal stem cell proliferation and differentiation. J Biosci Bioeng. 2008;105(6):586-594. doi:10.1263/jbb.105.586

14. Langenbach F, Handschel J. Effects of dexamethasone, ascorbic acid and $\beta$-glycerophosphate on the osteogenic differentiation of stem cells in vitro. Stem Cell Res Ther. 2013;4(5):117. doi:10.1186/scrt328

15. Brauer A, Pohlemann T, Metzger W. Osteogenic differentiation of immature osteoblasts: Interplay of cell culture media and supplements. Biotech Histochem. 2016;91(3):161169. doi:10.3109/10520295.2015.1110254

16. Dang A, Halloran B. High yield and high precision bone graft substitute from stem cells. Published online November 15, 2018:10. https://patents.google.com/patent/US20180326117A1

17. Gallagher JA, Gundle R, Beresford JN. Isolation and culture of bone-forming cells (osteoblasts) from human bone. Methods Mol Med. 1996;2:233-262. doi:10.1385/0-89603-335$\mathrm{X}: 233$

18. Devin CJ, Chotai S, McGirt MJ, et al. Intrawound vancomycin decreases the risk of surgical site infection after posterior spine surgery: a multicenter analysis. Spine (Phila $\mathrm{Pa}$ 1976). 2018;43(1):65-71. doi:10.1097/BRS.0000000000001371

19. Chu S, Chen N, Dang ABC, Kuo AC, Dang ABC. The effects of topical vancomycin on mesenchymal stem cells: more may not be better. Int J Spine Surg. 2017;11(2):12. doi:10. $14444 / 4012$
20. Bariteau JT, Kadakia RJ, Traub BC, Viggeswarapu M, Willett NJ. Impact of vancomycin treatment on human mesenchymal stromal cells during osteogenic differentiation. Foot Ankle Int. 2018;39(8):954-959. doi:10.1177/10711007187 66655

21. Dang ABC, Hong H, Lee K, Luan T, Reddy S, Kuo AC. Repurposing human osteoarthritic cartilage as a bone graft substitute in an athymic rat posterolateral spinal fusion model. Int J Spine Surg. 2018;12(6):735-742. doi:10.14444/5092

22. Kürüm B, Pekcan Z, Karsli B, Kumandaş A, Elma E. The effects of ketoprofen and meloxicam on bone healing in rat model: a comparative dual energy X-Ray absorptiometry study. Kafkas Universitesi Veteriner Fakultesi Dergisi. 2012;18(4):671676. doi:10.9775/kvfd.2012.6270

23. Inal S, Kabay S, Cayci MK, et al. Comparison of the effects of dexketoprofen trometamol, meloxicam and diclofenac sodium on fibular fracture healing, kidney and liver: an experimental rat model. Injury. 2014;45(3):494-500. doi:10. 1016/j.injury.2013.10.002

24. Pablos AB, Ramalho SA, König B, Furuse C, de Araújo VC, Cury PR. Effect of meloxicam and diclofenac sodium on peri-implant bone healing in rats. $J$ Periodontol. 2008;79(2):300-306. doi:10.1902/jop.2008.070301

25. Padayatty SJ, Sun H, Wang Y, et al. Vitamin C pharmacokinetics: implications for oral and intravenous use. Ann Intern Med. 2004;140(7):533-537. doi:10.7326/0003-4819140-7-200404060-00010

26. Jacob RA, Sotoudeh G. Vitamin C function and status in chronic disease. Nutr Clin Care. 2002;5(2):66-74. doi:10.1046/j. 1523-5408.2002.00005.x

Disclosures and COI: Drs Dang and Halloran report grants from Northern California Institute for Research and Education, during the conduct of the study, and have patent US20180326117A1 pending. Dr Dang reports grants from Johnson \& Johnson, outside the submitted work. No benefits in any form have been received or will be received from a commercial party related directly or indirectly to the subject of this article. All three authors, JC, KL, $\mathrm{KT}$, contributed equally to this study, and their names have been listed in alphabetical order by their last name.

Corresponding Author: Joshua Vic Chen, BA, 505 Parnassus Ave, San Francisco, CA 94143. Phone: (626) 235-7470; Email: joshua.chen2@ucsf. edu.

Published 3 December 2021

This manuscript is generously published free of charge by ISASS, the International Society for the Advancement of Spine Surgery. Copyright (c) 2021 ISASS. To see more or order reprints or permissions, see http://ijssurgery.com. 